\title{
ON COMPLEMENTED SUBSPACES OF SUMS AND PRODUCTS OF BANACH SPACES
}

\author{
M. I. OSTROVSKII
}

(Communicated by Dale Alspach)

\begin{abstract}
It is proved that there exist complemented subspaces of countable topological products (locally convex direct sums) of Banach spaces which cannot be represented as topological products (locally convex direct sums) of Banach spaces.
\end{abstract}

The problem of describing complemented subspaces of a given locally convex space is one of the general problems of the structure theory of locally convex spaces. In investigations of this problem in the particular case of spaces represented as countable products of Banach spaces (see [D1], [D2], [DO], [MM1]) the following problem arose (see [D2, p. 71], [MM2, p. 147]): Is every complemented subspace of a topological product (locally convex direct sum) of a countable family of Banach spaces isomorphic to a topological product (locally convex direct sum) of Banach spaces? G.Metafune and V.B.Moscatelli [MM3, p. 251] conjectured that this is false in general. The purpose of the present note is to prove this conjecture.

Our sources for basic concepts and results of Banach space theory and the theory of topological vector spaces are, respectively, $[\mathrm{LT}]$ and $[\mathrm{RR}]$.

Let us fix some terminology and notation. The algebra of all continuous linear operators on a Banach space $X$ will be denoted by $L(X)$. The identity mapping of a linear space $W$ is denoted by $I_{W}$. Let $\left\{X_{n}\right\}_{n=1}^{\infty}$ be a sequence of Banach spaces. We denote their Cartesian product endowed with the product topology by $\prod_{n=1}^{\infty} X_{n}$ and call it the topological product. We denote the locally convex direct sum of spaces $\left\{X_{n}\right\}_{n=1}^{\infty}$ by $\bigoplus_{n=1}^{\infty} X_{n}$. A linear subspace $Y$ of a topological vector space $Z$ will be called complemented if there is a continuous linear mapping $P$ of $Z$ onto $Y$ such that $P^{2}=P$. If $B$ is a subset of a linear space $V$, then the linear subspace of $V$ generated by $B$ will be denoted by $\operatorname{lin} B$. The dual of a locally convex space $Z$ endowed with its strong topology will be denoted by $Z_{\beta}^{\prime}$.

Theorem 1. A. There exist a sequence $\left\{X_{n}\right\}_{n=1}^{\infty}$ of Banach spaces and a complemented subspace $Y$ in $X=\bigoplus_{n=1}^{\infty} X_{n}$, such that $Y$ is not isomorphic to a locally convex direct sum of Banach spaces.

Received by the editors October 26, 1993 and, in revised form, December 1, 1994.

1991 Mathematics Subject Classification. Primary 46A04, 46A13; Secondary 47B99.

Key words and phrases. Complemented subspace, Banach space, topological product of Banach spaces, locally convex direct sum. 
B. There exist a sequence $\left\{Z_{n}\right\}_{n=1}^{\infty}$ of Banach spaces and a complemented subspace $W$ in $Z=\prod_{n=1}^{\infty} Z_{n}$, such that $W$ is not isomorphic to a topological product of Banach spaces.

Proof. First we shall prove part A. The main tool of our construction is the space with the property of bounded approximation but without $\pi$-property, constructed by C.J.Read $[R]$. Now we describe those details of Read's construction which we will use.

Let $V_{0}$ be a vector space of countable dimension with basis $\left\{e_{i}\right\}_{i=1}^{\infty}$. By $\mathbb{N}_{6}$ we denote the set $\mathbb{N} \backslash\{1,2,3,4,5\}$. Let $a_{r}=5 \cdot 10^{r-2}, b_{r}=10^{r}, r \in \mathbb{N}_{6}$ and $V=\operatorname{lin}\left\{e_{i}: i>a_{6}\right\}$. Let us introduce in $V$ three collections of finite-dimensional subspaces:

$$
\begin{gathered}
V_{r}=\operatorname{lin}\left\{e_{i}: a_{r}<i \leq b_{r}\right\}, r \in \mathbb{N}_{6}, \\
W_{r}=\operatorname{lin}\left\{e_{i}: a_{r+1}<i \leq b_{r}\right\}=V_{r} \cap V_{r+1}, r \in \mathbb{N}_{6}, \\
U_{r}=\left\{\begin{array}{l}
\operatorname{lin}\left\{e_{i}: b_{r-1}<i \leq b_{r}\right\}, r \in \mathbb{N}_{6} \backslash\{6\}, \\
V_{6}, r=6 .
\end{array}\right.
\end{gathered}
$$

We introduce the notation

$$
E(r)=\left\{\begin{array}{l}
\left\{i: b_{r-1}<i \leq b_{r}\right\}, r \in \mathbb{N}_{6} \backslash\{6\} \\
\left\{i: a_{6}<i \leq b_{6}\right\}, r=6
\end{array}\right.
$$

By $\pi_{r}^{V}: V \rightarrow V_{r}, \pi_{r}^{W}: V \rightarrow W_{r}$, and $\pi_{r}^{U}: V \rightarrow U_{r}$ we denote natural projections. For example,

$$
\pi_{r}^{V}\left(\sum_{i>a_{6}} d_{i} e_{i}\right)=\sum_{a_{r}<i \leq b_{r}} d_{i} e_{i} .
$$

Given some norms $\left\{\|\cdot\|_{(r)}\right\}_{r=6}^{\infty}$ on $V_{r}$, we endow $V$ with the norm

$$
\|x\|^{2}=\sum_{r=6}^{\infty}\left[\left\|\pi_{r}^{V}(x)\right\|_{(r)}^{2}+\frac{1}{\log r}\left(\left\|\pi_{r}^{W}(x)\right\|_{(r)}^{2}+\left\|\pi_{r}^{W}(x)\right\|_{(r+1)}^{2}\right)\right] .
$$

Let $R$ be the completion of $V$ under the norm $\|\cdot\|$.

Let $A$ be a subset of $\mathbb{N}_{6}$. We denote the closure in the norm topology of the subspace of $R$ spanned by the vectors

$$
\left\{e_{i}: i \in \bigcup_{j \in A} E(j)\right\}
$$

by $R_{A}$. The arguments of C.J.Read [R] imply the following result.

Theorem 2. For some collection of norms $\|\cdot\|_{(r)}\left(r \in \mathbb{N}_{6}\right)$, there exist a convergentto-zero sequence of positive numbers $\{\varepsilon(r)\}_{r=6}^{\infty}$ and numbers $0<\alpha, \beta<\infty$ such that for every $A \subset \mathbb{N}_{6}$ and every $j \in A$ there exists a mapping $\xi_{j}: L\left(R_{A}\right) \rightarrow \mathbb{R}$ for which the following conditions are satisfied:

(a) If $\left.\pi_{j}^{W} T\right|_{W_{j}}=\mu I_{W_{j}}$, then $\xi_{j}(T)=\mu$.

(b) $\left|\xi_{j}(T S)-\xi_{j}(T) \xi_{j}(S)\right| \leq \alpha j^{-\beta}|| T|||| S||$.

(c) If $j, j+1 \in A$, then $\left|\xi_{j+1}(T)-\xi_{j}(T)\right| \leq \varepsilon(j+1)\|T\|$. 
Starting from this point we suppose that $R$ is the space constructed by the described method using some collection of norms $\left\{\|\cdot\|_{(r)}\right\}_{r=6}^{\infty}$ satisfying the conditions of Theorem 2. The precise description of the norms $\|\cdot\|_{(r)}$ does not matter for us. We shall use only the definition of $\|\cdot\|$ and the properties of the spaces $R_{A}$ listed in Theorem 2.

Let $f: \mathbb{N}_{6} \rightarrow \mathbb{R}$ be some function. Each vector $v \in V$ can be represented in a unique manner as follows:

$$
v=\sum_{j=6}^{\infty} \sum_{i \in E(j)} d_{i} e_{i}
$$

Therefore the formula

$$
M_{f} v=\sum_{j=6}^{\infty} \sum_{i \in E(j)} f(j) d_{i} e_{i}
$$

defines a linear mapping on $V$.

Lemma 1. If the function $f$ is such that for some $C<\infty$ and each $k \in \mathbb{N}_{6}$ the conditions

$$
\begin{gathered}
|f(k)| \leq C, \\
|f(k)-f(k+1)| \leq C / \sqrt{\log k}
\end{gathered}
$$

are satisfied, then $M_{f}$ is bounded with respect to the norm $\|\cdot\|$ and $\left\|M_{f}\right\| \leq 2 C$.

Proof. Let us note that for every $v \in V$ we have

$$
\begin{gathered}
\pi_{6}^{V} M_{f} v=f(6) \pi_{6}^{V} v \\
\pi_{r}^{V} M_{f} v=f(r) \pi_{r}^{V} v+(f(r-1)-f(r)) \pi_{r-1}^{W} v, r \in \mathbb{N}_{6} \backslash\{6\} \\
\pi_{r}^{W} M_{f} v=f(r) \pi_{r}^{W} v, r \in \mathbb{N}_{6} .
\end{gathered}
$$

Hence, we have

$$
\begin{aligned}
\left\|M_{f} v\right\|^{2}= & \sum_{r=6}^{\infty}\left[\left\|\pi_{r}^{V} M_{f} v\right\|_{(r)}^{2}+\frac{1}{\log r}\left(\left\|\pi_{r}^{W} M_{f} v\right\|_{(r)}^{2}+\left\|\pi_{r}^{W} M_{f} v\right\|_{(r+1)}^{2}\right)\right] \\
\leq & |f(6)|^{2}\left\|\pi_{6}^{V} v\right\|_{(6)}^{2}+\sum_{r=7}^{\infty}\left(\left|f(r)\|\| \pi_{r}^{V} v\left\|_{(r)}+\mid f(r)-f(r-1)\right\|\left\|\pi_{r-1}^{W} v\right\|_{(r)}\right)^{2}\right. \\
& +\sum_{r=6}^{\infty}|f(r)|^{2} \frac{1}{\log r}\left(\left\|\pi_{r}^{W} v\right\|_{(r)}^{2}+\left\|\pi_{r}^{W} v\right\|_{(r+1)}^{2}\right) \\
\leq & C^{2}\left\|\pi_{6}^{V} v\right\|_{(6)}^{2}+\sum_{r=7}^{\infty} 2 C^{2}\left\|\pi_{r}^{V} v\right\|_{(r)}^{2}+\sum_{r=7}^{\infty} \frac{2 C^{2}}{\log (r-1)}\left\|\pi_{r-1}^{W} v\right\|_{(r)}^{2} \\
& +\sum_{r=6}^{\infty} \frac{C^{2}}{\log r}\left(\left\|\pi_{r}^{W} v\right\|_{(r)}^{2}+\left\|\pi_{r}^{W} v\right\|_{(r+1)}^{2}\right) \\
\leq & 4 C^{2}\|v\|^{2} .
\end{aligned}
$$

The required inequality follows. 
Therefore, if the conditions of Lemma 1 are satisfied, then $M_{f}$ may be considered as an element of $L(R)$.

Let $\left\{f_{n}\right\}_{n=1}^{\infty}$ be a sequence of functions satisfying the conditions of Lemma 1 . Introduce the notation $M_{n}:=M_{f_{n}} ; A(n):=\operatorname{supp} f_{n} \subset \mathbb{N}_{6}$.

Lemma 2. There exists a sequence $\left\{f_{n}\right\}_{n=1}^{\infty}$ such that

(a) $\sup _{n}\left\|M_{n}\right\|<\infty$.

(b) If $j \in A(n)$, then $f_{n+1}(j)=1$.

(c) For every $j \in \mathbb{N}_{6}$ there exists $m \in \mathbb{N}$ such that $f_{m}(j)=1$.

(d) For every $n \in \mathbb{N}$ and every $r \in \mathbb{N}_{6}$ there exists $j>r$ such that $j \in A(1)$ and there exists $i>j$ such that $i \notin A(n)$ and $\{j, j+1, \ldots, i\} \subset A(n+1)$.

Proof. Let $\{p(s)\}_{s=1}^{\infty} \subset \mathbb{N}_{6}$ be an increasing sequence of natural numbers such that

$$
\sum_{k=p(s)}^{p(s+1)-1} 1 / \sqrt{\log k} \geq 1 .
$$

Let $\{s(m)\}_{m=1}^{\infty}$ be the sequence defined by the equality

$$
s(m)=1+2+3+\ldots+m=m(m+1) / 2 .
$$

Let $A(n)$ be the sets represented as unions of intervals of integers in the following way:

$$
A(n)=\bigcup_{m=1}^{\infty}\{p(s(m)-n)+1, \ldots, p(s(m)+n)-1\} .
$$

If $s(m)-n<1$ we let $p(s(m)-n)=5$. From the definitions of the sequences $\{p(s)\}_{s=1}^{\infty}$ and $\{s(m)\}_{m=1}^{\infty}$ it follows that there exists a sequence $\left\{f_{n}\right\}_{n=1}^{\infty}$ of functions, $f_{n}: \mathbb{N}_{6} \rightarrow \mathbb{R}$, satisfying the following conditions:

(e) $\left(\forall k \in \mathbb{N}_{6}\right)\left(0 \leq f_{n}(k) \leq 1\right)$;

(f) $\operatorname{supp} f_{n}=A(n)$;

(g) $\left\{k: f_{1}(k)=1\right\}=\{p(s(m))\}_{m=1}^{\infty}$;

(h) $\left\{k: f_{n}(k)=1\right\}=A(n-1)(n \geq 2)$;

(i) $\left|f_{n}(k)-f_{n}(k+1)\right| \leq 1 / \sqrt{\log k}$.

By Lemma 1 the conditions (e) and (i) imply (a). It is clear that the conditions (f)-(h) imply (b) and (c).

In order to verify condition (d) we let $j$ be any element of the sequence

$$
\{p(s(m))\}_{m=1}^{\infty},
$$

$j=p(s(k))$, for which $j>r$ and $k>2 n$. Let $i=p(s(k)+n)$. By the conditions (f) and (g) it follows that $i$ and $j$ satisfy condition (d). Lemma 2 is proved.

It is clear that the conditions (a), (b) and (c) of Lemma 2 imply that the sequence $\left\{M_{n}\right\}_{n=1}^{\infty}$ converges to the identity operator in the strong operator topology.

Let $C(n)=A(n) \backslash A(n-1)$ (we set $A(0)=\emptyset)$. Set

$$
G=R \oplus_{p}\left(\sum_{n=1}^{\infty} \oplus R_{C(n)}\right)_{p},
$$

where $1<p<\infty$ is an arbitrary number. 
We recall that by the definitions of direct sums, $G$ is the space of those sequences of the form

$$
g=\left(x, y_{1}, y_{2}, \ldots, y_{n}, \ldots\right),
$$

for which $x \in R, y_{n} \in R_{C(n)}$ and

$$
\|x\|^{p}+\sum_{n=1}^{\infty}\left\|y_{n}\right\|^{p}<\infty
$$

Linear operations on $G$ are defined in a coordinatewise manner, and the norm is defined by the formula

$$
\|g\|=\left(\|x\|^{p}+\sum_{n=1}^{\infty}\left\|y_{n}\right\|^{p}\right)^{1 / p} .
$$

We introduce operators $S_{n} \in L(G)(n \in \mathbb{N})$ by the formulas

$$
\begin{aligned}
& S_{n}\left(x, y_{1}, y_{2}, \ldots, y_{n}, \ldots\right) \\
& \quad=\left(M_{n} x+y_{n}, y_{1}, \ldots, y_{n-1},\left(M_{n}-M_{n}^{2}\right) x+\left(I-M_{n}\right) y_{n}, 0,0, \ldots\right) .
\end{aligned}
$$

These operators are well defined because by condition (b) of Lemma 2 the image of $\left(M_{n}-M_{n}^{2}\right)$ is contained in $R_{C(n)}$.

By direct verification it follows that the sequence $\left\{S_{n}\right\}_{n=1}^{\infty}$ is uniformly bounded, $S_{n}$ are projections and

$$
S_{n} S_{m}=S_{\min (m, n)}(m, n \in \mathbb{N})
$$

Furthermore,

$$
(\forall g \in G)\left(\lim _{n \rightarrow \infty} S_{n} g=g\right),
$$

where the limit is taken in the norm topology. Therefore $\left\{S_{n}\right\}_{n=1}^{\infty}$ generates a Schauder decomposition of $G$.

The construction above is taken from [CK], it goes back to W.B.Johnson [J].

The sequence $\left\{S_{n} G\right\}_{n=1}^{\infty}$ is an increasing sequence of subspaces of $G$. Let $X$ be a strict inductive limit of this sequence. Since each $S_{n-1} G$ is complemented in $S_{n} G$, $X$ is isomorphic to a locally convex direct sum of a sequence of Banach spaces. Since $X=\bigcup_{n=1}^{\infty} S_{n} G, X$ may be considered as a subset and even a linear subspace of $G$.

Let us denote by $P$ the projection on $G$ defined by the formula

$$
P\left(x, y_{1}, y_{2}, \ldots, y_{n}, \ldots\right)=(x, 0,0, \ldots, 0, \ldots) .
$$

By definition of the operator $S_{n}$ it follows that

$$
S_{n} G \subset R_{A(n)} \oplus_{p}\left(\sum_{k=1}^{n} \oplus R_{C(k)}\right)_{p} \oplus_{p}\left(\sum_{k=n+1}^{\infty} \oplus\{0\}\right)_{p} .
$$


By condition (b) of Lemma 2 the restriction of $M_{n}(n \geq 2)$ to $R_{A(n-1)}$ coincides with the identity operator. Therefore

$$
S_{n} G \supset R_{A(n-1)} \oplus_{p}\left(\sum_{k=1}^{n-1} \oplus R_{C(k)}\right)_{p} \oplus_{p}\left(\sum_{k=n}^{\infty} \oplus\{0\}\right)_{p} .
$$

Therefore $P S_{n} G \subset S_{n+1} G$ and $P$ may be considered also as a projection on $X$. Let $Y=P X$. We claim that

(I) $P$ is a continuous projection on $X$.

(II) $Y$ considered as a subspace of $X$ is not isomorphic to a locally convex direct sum of Banach spaces.

The topologies of $X$ and $G$ coincide on each of $S_{n} G$ [RR, p.127]. Since $X$ is a strict inductive limit of $\left\{S_{n} G\right\}_{n=1}^{\infty}$, then in order to prove statement (I), it is sufficient to prove that the restriction of $P$ to each $S_{n} G$ is continuous [RR, p.79]. But this immediately follows from the inclusion $P S_{n} G \subset S_{n+1} G$ and the continuity of $P$ considered as a mapping from $G$ to $G$ (with its initial Banach topology).

Let us prove (II). We have

$$
Y=\bigcup_{n=1}^{\infty} P S_{n} G
$$

Therefore

$$
R_{A(n-1)} \oplus_{p}\left(\sum_{k=1}^{\infty} \oplus\{0\}\right)_{p} \subset Y \cap S_{n} G \subset R_{A(n)} \oplus_{p}\left(\sum_{k=1}^{\infty} \oplus\{0\}\right)_{p} .
$$

Hence

$$
Y=\bigcup_{n=1}^{\infty}\left(R_{A(n)} \oplus_{p}\left(\sum_{k=1}^{\infty} \oplus\{0\}\right)_{p}\right) .
$$

Let us show that the topology on $Y$ induced by the strict inductive topology of $X$ coincides with the topology of the strict inductive limit of the sequence

$$
\left\{R_{A(n)} \oplus_{p}\left(\sum_{k=1}^{\infty} \oplus\{0\}\right)_{p}\right\}_{n=1}^{\infty} .
$$

We denote

$$
R_{A(n)} \oplus_{p}\left(\sum_{k=1}^{\infty} \oplus\{0\}\right)_{p}
$$

by $K_{n}(n \in \mathbb{N})$.

From (2) it follows that the intersection of an arbitrary neighbourhood of zero in $X$ with $K_{n-1}$ contains a neighbourhood of zero in $K_{n-1}$. Hence the topology induced on $Y$ from $X$ is not stronger than the strict inductive topology of the sequence (3).

On the other hand, let $\tau$ be a convex neighbourhood of zero in the strict inductive limit of the sequence (3). Then $\tau_{n}:=\tau \cap K_{n}$ is a neighbourhood of zero in $K_{n}$. Let

$$
\theta_{n}:=\left\{\left(x, y_{1}, \ldots, y_{n}, \ldots\right) \in G:(x, 0, \ldots, 0, \ldots) \in \tau_{n}\right\} .
$$


We have $\theta_{n} \cap Y=\tau_{n}$. From (1) it follows that $\sigma_{n}:=\theta_{n} \cap S_{n} G$ is a neighbourhood of zero in $S_{n} G$. It is also clear that $\left\{\sigma_{n}\right\}_{n=1}^{\infty}$ is an increasing sequence of convex sets. Therefore $\sigma:=\bigcup_{n=1}^{\infty} \sigma_{n}$ is a neighbourhood of zero in $X$. It is easy to see that $\sigma \cap Y \subset \tau$. Hence the topology of the strict inductive limit of the sequence (3) is not stronger than the topology induced on $Y$ from $X$. Hence these topologies coincide.

So it remains to prove that the strict inductive limit of the sequence $\left\{R_{A(n)}\right\}_{n=1}^{\infty}$ is not isomorphic to a locally convex direct sum of Banach spaces.

Assume the contrary. Let us denote the strict inductive limit of $\left\{R_{A(n)}\right\}_{n=1}^{\infty}$ by $S$, and let $S=\bigoplus_{\lambda \in \Lambda} S_{\lambda}$, where $S_{\lambda}$ are Banach spaces. Since the space $R_{A(1)}$ is a Banach space, it has a bounded neighbourhood of zero. By the description of bounded sets in locally convex direct sums [RR, p.92] it follows that for some finite subset $\Delta \subset \Lambda$ the space $R_{A(1)}$ is contained in

$$
T=\left(\bigoplus_{\lambda \in \Delta} S_{\lambda}\right) \oplus\left(\bigoplus_{\lambda \in \Lambda \backslash \Delta}\{0\}\right)
$$

The space $T$ is isomorphic to a Banach space. Hence it has a bounded neighbourhood of zero. By the description of bounded sets in strict inductive limits $[R R$, p.129] it follows that $T$ is contained in $R_{A(k)}$ for some $k \in \mathbb{N}$.

It is clear that $T$ is complemented in $S$. Hence $T$ is complemented in $R_{A(j)}$ for every $j \geq k$. Let $Q \in L\left(R_{A(k+1)}\right)$ be some projection onto $T$. Let $r \in \mathbb{N}_{6}$ be such that for every $s \geq r$ we have $\varepsilon(s)\|Q\|<1 / 10$ and $\alpha s^{-\beta}\|Q\|^{2}<1 / 100$. Let $i$ and $j$ be natural numbers satisfying condition (d) of Lemma 2 for given $r$ and for $n=k$. Since $R_{A(1)}$ is in the image of the operator $Q$ and $j \in A(1)$, by condition (a) of Theorem 2 it follows that $\xi_{j}(Q)=1$. Since the image of $Q$ is in $R_{A(k)}$ and $i \notin A(k)$, we have $\pi_{i}^{W} Q=0$. So by condition (a) of Theorem 2 it follows that $\xi_{i}(Q)=0$.

Since we have $\{j, j+1, \ldots, i\} \subset A(k+1)$ (condition (d) of Lemma 2), by parts (b) and (c) of Theorem 2 we have

$$
(\forall q \in\{j, \ldots, i-1\})\left(\left|\xi_{q}(Q)-\xi_{q+1}(Q)\right|<1 / 10\right)
$$

and

$$
(\forall q \in\{j, \ldots, i\})\left(\left|\xi_{q}(Q)-\xi_{q}^{2}(Q)\right|<1 / 100\right) .
$$

The last assertion implies that either $\left|\xi_{q}(Q)\right|<1 / 10$ or $\left|\xi_{q}(Q)\right|>9 / 10$. We have arrived at a contradiction. Hence $S$ is not isomorphic to a locally convex direct sum of Banach spaces. Part A of Theorem 1 is now proved.

Let us turn to part B.

Let

$$
H=\left(\sum_{r=6}^{\infty} \oplus\left(V_{r},\|\cdot\|_{(r)}\right)\right)_{2} \oplus_{2}\left(\sum_{r=6}^{\infty} \oplus\left(W_{r},\|\cdot\|_{(r)}\right)\right)_{2} \oplus_{2}\left(\sum_{r=6}^{\infty} \oplus\left(W_{r},\|\cdot\|_{(r+1)}\right)\right)_{2} .
$$

From the description of the Banach space $R$ it is clear that the mapping $T: R \rightarrow H$ defined as

$$
T x=\left(\left\{\pi_{r}^{V}(x)\right\}_{r=6}^{\infty}, \frac{1}{\sqrt{\log r}}\left\{\pi_{r}^{W}(x)\right\}_{r=6}^{\infty}, \frac{1}{\sqrt{\log r}}\left\{\pi_{r}^{W}(x)\right\}_{r=6}^{\infty}\right)
$$


is an isometric embedding. Because the spaces $V_{r}$ and $W_{r}$ are finite dimensional, it follows from the well known description of the duals of direct sums that $H$ is reflexive. Hence $R$ is reflexive. By the construction of $G$ it follows that $G$ is also a reflexive Banach space. By the well-known description of duals of locally convex direct sums and topological products [RR, p.93] it follows that the space $X$ is also reflexive. Let $Z=X_{\beta}^{\prime}$. From the description of a dual of $X$ [RR, p.93] and from the description of bounded sets of $X$ [RR, p.92] it follows that $Z$ is isomorphic to a countable topological product of Banach spaces.

Let $P^{\prime}$ be the adjoint mapping of $P$. It is well known [RR, p.48] that $P^{\prime}$ is continuous in $Z$. Hence $Z$ is isomorphic to a direct sum

$$
W \oplus U
$$

where $W=P^{\prime} Z$ and $U=\left\{z \in Z: P^{\prime} z=0\right\}$ (see [RR, p.96]). Hence

$$
Z_{\beta}^{\prime}=W_{\beta}^{\prime} \oplus U_{\beta}^{\prime},
$$

where $W_{\beta}^{\prime}$ is the image of $P^{\prime \prime}: Z_{\beta}^{\prime} \rightarrow Z_{\beta}^{\prime}$. But, because of reflexivity, $Z_{\beta}^{\prime}=X$ and $P^{\prime \prime}=P$. Hence $Y=W_{\beta}^{\prime}$.

So if we suppose that $W$ is isomorphic to a topological product of Banach spaces, then by description of duals of topological products [RR, p.93] it would follow that $Y$ is isomorphic to a locally convex direct sum of Banach spaces.

Part B of Theorem 1 is proved.

\section{REFERENCES}

[CK] P.G.Casazza and N.J.Kalton, Notes on approximation properties in separable Banach spaces, Geometry of Banach Spaces (Strobl, 1989), London Math. Soc. Lecture Note Ser., 158 (P.Müller and W.Schachermayer, eds.), Cambridge University Press, 1990, pp. 49-63. MR 92d: 46022

[D1] P.Domański, Complemented subspaces of products of Hilbert spaces, Proc. Amer. Math. Soc. 110 (1990), 187-196. MR 90m:46007

[D2], $\mathcal{L}_{p}$-spaces and injective locally convex spaces, Rozprawy Mat. 298 (1990), 1-76. MR 91j: 46002

[DO] P.Domański and A.Ortyński, Complemented subspaces of products of Banach spaces, Trans. Amer. Math. Soc. 316 (1989), 215-231. MR 90b:46004

[J] W.B.Johnson, Factoring compact operators, Israel J. Math. 9 (1971), 337-345. MR 44:7318

[LT] J.Lindenstrauss and L.Tzafriri, Classical Banach spaces, v. I, Springer-Verlag, Berlin Heidelberg New York, 1977. MR 58:17766

[MM1] G.Metafune and V.B.Moscatelli, Complemented subspaces of sums and products of Banach spaces, Ann. Mat. Pura Appl. 153 (1989), 1-16.

[MM2] - On twisted Fréchet and (LB)-spaces, Proc. Amer. Math. Soc. 108 (1990), 145150. MR 90d: 46007

[MM3] _ Quojections and prequojections, Advances in the Theory of Fréchet spaces (T.Terzioğlu, ed.), Kluwer Academic Publishers, Dordrecht, 1989, pp. 235-254. MR 92e:46006

[R] C.J.Read, Different forms of the approximation property (to appear).

[RR] A.P.Robertson and W.Robertson, Topological vector spaces, Cambridge University Press, Cambridge, 1973. MR 50:2854

Mathematical Division, Institute for Low Temperature Physics and Engineering, 47 Lenin aVenue, 310164 Kharkov, Ukraine

E-mail address: mostrovskii@ilt.kharkov.ua 